\title{
On the statistical implication of timing noise for pulsar braking index
}

\author{
A. E. Chukwude $1,2, \star$ \\ ${ }^{1}$ Hartebeesthoek Radio Astronomy Observatory, PO Box 443, Krugersdorp 1740, South Africa \\ e-mail: austine@hartrao.ac.za \\ 2 Department of Physics \& Astronomy, University of Nigeria, Nsukka, Enugu State, Nigeria \\ e-mail: aus_chukwude@yahoo.com
}

Received 24 February 2003 / Accepted 13 May 2003

\begin{abstract}
Timing data on 27 radio pulsars spanning more than 13 years were analysed in order to investigate the correlations of radio pulsar timing noise (random fluctuations in the observed pulse phase - RPTN), with the observed electromagnetic torque braking index $\left(n_{\mathrm{obs}}\right)$. The results reveal significant correlations $(r \geq 90 \%)$ between the statistics used to parameterize RPTN and the absolute magnitude of the observed second time derivative of the pulse (rotation) frequency ( $\left.\ddot{v}_{\text {obs }}\right)$. These correlations, most plausibly, suggest that the observed braking indices of most pulsars, obtained through the traditional phase-connected method, are strongly dominated by intrinsic variability in their spin rates. The implication of this result for a significant measurement of pulsar systematic frequency second derivative is discussed.
\end{abstract}

Key words. methods: statistical - stars: neutron - pulsars: general

\section{Introduction}

Radio pulsars are widely accepted to be rapidly spinning, highly magnetized neutron stars. The rotational kinetic energy of such stars powers their pulsed electromagnetic radiation, in addition to accelerating particle winds. In the standard dipole radiation model, which describes this conversion of rotational kinetic energy into vacuum magnetic dipole radiation (e.g. Goldreich \& Julian 1969), the braking of an isolated pulsar follows a simple power-law relation of the form

$\dot{v}=-K v^{n}$

where $v$ is the pulsar rotation frequency, $\dot{v}$ is its first derivative with respect to time, $n$ is the braking index and $K$ is assumed to be an arbitrary positive constant and depends explicitly on the inertial and magnetic dipole moments of the neutron star. For the highly simplified model, in which the pulsar spin-down torque arises solely from magnetic dipole radiation at the spin frequency, the braking index $n=3$. In terms of the pulsar rotational observables, $n$ is obtained (e.g. Manchester \& Taylor 1977) from

$n=\frac{v \ddot{v}}{\dot{v}^{2}}$,

where $\ddot{v}$ is the second derivative of the rotation frequency with respect to time.

\footnotetext{
* e-mail: aus_chukwude@yahoo.com
}

However, long-term timing observations of radio pulsars have revealed enormous complexities in the true dynamical behaviour of most pulsars. For instance, it is now widely believed that a variety of rotational irregularities, in the form of timing noise and glitches - sudden changes (the larger ones exclusively increases) in the magnitudes of pulsar spin rates - is superimposed on the systematic pulsar spin down. While glitches have been observed in just a few radio pulsars (Wang et al. 2000 and references therein), timing noise - sustained, random fluctuations in the observed pulse phase - is more prevalent among the known pulsar population (Boynton et al. 1972; Cordes \& Downs 1985; Chukwude 2002). In principle, a qualitative assessment of the level of timing noise activity for a given pulsar can be obtained from the ("excess") phase residuals remaining after accounting for its deterministic spindown (Cordes \& Greenstein 1981; Cordes \& Downs 1985; Cordes 1993; D’Alessandro et al. 1995). A statistic of importance is the timing noise activity parameter $(A)$ defined, following Cordes \& Downs (1985), as

$A=\log \left[\frac{\sigma_{\mathrm{TN}}(m, T)}{\sigma_{\mathrm{TN}}(m, T)_{\mathrm{crab}}}\right]$,

where $m$ is the order of the polynomial fit to the data, $T$ is the time span of the fit in days, $\sigma_{\mathrm{TN}}(m, T)$ is the timing noise contribution to the gross rms phase residuals, in $\mathrm{ms}$, and $\sigma_{\mathrm{TN}}(m, T)_{\text {crab }}$ is the timing noise of the Crab pulsar in ms, which scales with $T$ as $\sigma_{\mathrm{TN}}(m, T)_{\text {crab }}=12(T / 1628)^{3 / 2}$. The scaling of the Crab pulsar timing noise activity with $T$ is 
consistent with frequency noise (Groth 1975; Cordes 1980; Cordes \& Helfand 1980; Cordes 1993).

It has now been widely observed (Boynton et al. 1972; D'Alessandro et al. 1993; Chukwude 2002) that the $m=3$ polynomial model appears to describe the observations of most pulsars significantly better than the $m=2$ model, in that both the rms phase residuals and the reduced chi-squares are significantly less for the third-order model. However, there is still controversy over whether the $m=3$ polynomial models offer a better description of the intrinsic pulsar spin-down instead of just merely absorbing fluctuations in the pulsar spin rates. The coefficient of the cubic term obtained from this phaseconnected solution will be referred to throughout this paper as the observed frequency second derivative $\left(\ddot{v}_{\text {obs }}\right)$. In view of the complicated nature of radio pulsar spin evolution, the observed frequency second derivative can be expressed as

$\ddot{v}_{\text {obs }}=\ddot{v}_{\text {tno }}+\ddot{v}_{\text {pre }}$,

where $\ddot{v}_{\text {tno }}$ is the contribution from all forms of fluctuations (including unresolved glitches) in the pulsar rotation rates and $\ddot{v}_{\text {pre }}$ is the contribution from the intrinsic pulsar torque braking processes. Assuming 1) that the third-order polynomial is only modelling the intrinsic pulsar rotation history ( $\ddot{v}_{\text {pre }} \gg \ddot{v}_{\text {tno }}$ and $\ddot{v}_{\text {obs }} \approx \ddot{v}_{\text {pre }}$ ) and 2 ) the pulsar's spin brakes via purely electromagnetic torques, the observed frequency second derivative is expected to yield $n_{\mathrm{obs}} \approx 3$. Alternately, if the coefficient of the cubic term merely absorbs fluctuations in the pulsar spin rates, i.e. $\ddot{v}_{\text {pre }} \ll\left|\ddot{v}_{\text {tno }}\right|$, then $\ddot{v}_{\text {obs }} \approx \ddot{v}_{\text {tno. }}$. In this case the resulting $\ddot{v}_{\text {obs }}$ would be expected to be highly variable, with the sign of $\ddot{v}_{\text {tno }}$ being either positive or negative, thus yielding anomalous braking indices $\left(n_{\text {obs }}\right)$ whose absolute values could deviate significantly from the canonical value of 3 . Furthermore, one would expect a significant correlation between the absolute value of the observed frequency second derivative $\left(\ddot{v}_{\text {obs }}\right)$ and any statistic used to quantify the rotational fluctuations which, presumably, would be absorbed by the coefficient of the cubic term. Accordingly, we define

$\sigma_{\mathrm{R} 23}=\sigma_{\mathrm{R}}(2, T)-\sigma_{\mathrm{R}}(3, T)$

as a statistical measure of the amount of phase fluctuations absorbed by the coefficient of the cubic term, where $\sigma_{\mathrm{R}}(2, T)$ and $\sigma_{\mathrm{R}}(3, T)$ are the rms phase residuals obtained from secondand third-order polynomials, respectively, fitted over a time span $T$. Currently, there appears to be some consensus that the observed $\ddot{v}$ for most pulsars is severely biased by fluctuations of timing noise origin, a situation which precludes significant measurements of $n$ for most radio pulsars (Baykal et al. 1999; Johnston \& Galloway 1999). However, the exact form of the dependence of $n$ on radio pulsar timing noise is not yet well understood.

In this paper, radio timing data on 27 pulsars, collected at Hartebeesthoek Radio Astronomy Observatory (HartRAO), are analysed in order to investigate the forms of statistical dependence of $\ddot{v}_{\text {obs }}$ on the observed timing noise activity, as parameterized by the statistics $A$ and $\sigma_{\mathrm{R} 23}$.

\section{Observations and data analyses}

Timing observations of radio pulsars at Hartebeesthoek Radio Astronomy Observatory in South Africa commenced 1984 and are still on-going at the time of this writing. However, continuous pulsar observations were interrupted between June 1999 and December 2000. Consequently, the data reported here are those accumulated between January 1984 and May 1999. Observations were made at regular intervals of $\sim 14$ days at either 1668 or $2272 \mathrm{MHz}$ using the 26-m HartRAO radio telescope. Pulses were recorded through a single $10 \mathrm{MHz}$ bandwidth receiver at both frequencies and no pre-detection dedispersion hardware was available. Detected pulses were smoothed with an appropriate time constant, and integrated over $N_{\mathrm{p}}$ consecutive rotation periods, where $N_{\mathrm{p}}$ is different for different pulsars. Integration commenced at a particular second via synchronization to the station clock, which is derived from a hydrogen maser and is referenced to UTC via GPS.

All topocentric arrival times obtained at HartRAO were transformed to infinite observing frequency at the Solar System Barycenter (SSB) using the Jet Propulsion Laboratory DE200 solar system ephemeris and the TEMPO software package (http://pulsar.princeton.edu/tempo). The barycentric arrival times were subsequently modelled using the HartRAO in-house timing analysis software, which is based on the standard pulsar timing technique of Manchester \& Taylor (1977) as described in Flanagan (1995). The time evolution of the rotational phase of a non-binary pulsar (when corrected to the SSB) can be approximated by a polynomial of the form (Lyne \& Graham-Smith 1998):

$\phi(t)=\phi_{0}+v\left(t-t_{0}\right)+\frac{1}{2} \dot{v}\left(t-t_{0}\right)^{2}+\frac{1}{6} \ddot{v}\left(t-t_{0}\right)^{3}$,

where $\phi_{0}$ is the phase at an arbitrary time epoch $t_{0}, v, \dot{v}$ and $\ddot{v}$ are the rotation frequency, and its first and second time derivatives, respectively. In practice, for sufficiently accurate values of $v$, $\dot{v}$ and $\ddot{v}$, Eq. (3) can be used to predict the phase/(Barycentric time of pulse peak arrival, BTOA) of a given pulsar at/(very near) any time $t$. The phase residuals, $\mathfrak{R}\left(t_{\mathrm{j}}\right)$ for $1 \leq j \leq N$ where $N$ is the number of observations for a given pulsar, are given by the difference between the observed and predicted BTOA's. $\mathfrak{R}\left(t_{\mathrm{j}}\right)$ are used to calculate the root-meansquare phase residuals $\sigma_{\mathrm{R}}(m, T)$ (where, e.g., $m=2$ is order of the polynomial) and the rms "white noise" $\sigma_{\mathrm{W}}(T)$, following Cordes \& Downs (1985). Pulse-to-pulse phase jitter, measurement uncertainty, and other effects that fluctuate on much shorter timescales ( $<1$ day), all contribute to the white noise component. The rms timing noise $\sigma_{\mathrm{TN}}$ is calculated as: $\sigma_{\mathrm{TN}}=\left\{\sigma_{\mathrm{R}}^{2}(2, T)-\sigma_{\mathrm{W}}^{2}(T)\right\}^{1 / 2}$.

\section{Results}

The results of the data analysis for 27 HartRAO pulsars are summarized in Table 1. Column 1 lists the name of the pulsar, using the B1950.0 naming convention; Cols. 2 and 3, respectively, give the rms phase residuals from $m=2$ and 3 polynomial models; Col. 4 lists the timing noise statistic $\sigma_{\mathrm{R} 23} ;$ Col. 5 shows the ratio of the reduced chi-squares of the second- and 
Table 1. Results of the second- and third-order polynomial fits to the 27 HartRAO pulsars.

\begin{tabular}{|c|c|c|c|c|c|c|c|c|c|c|}
\hline $\begin{array}{l}\text { Object } \\
P S R B \\
(1)\end{array}$ & $\begin{array}{r}\sigma_{\mathrm{R}}(2, T) \\
(m P) \\
(2)\end{array}$ & $\begin{array}{r}\sigma_{\mathrm{R}}(3, T) \\
(m P) \\
(3)\end{array}$ & $\begin{array}{r}\sigma_{\mathrm{R} 23} \\
(m P) \\
(4)\end{array}$ & $\begin{array}{l}\chi_{23}^{2} \\
(5)\end{array}$ & $\begin{array}{c}T \\
\text { (Days) } \\
\text { (6) }\end{array}$ & $\begin{array}{c}\ddot{v}_{\mathrm{obs}} \\
10^{-25} \mathrm{~Hz} \mathrm{~s}^{-2} \\
\text { (7) }\end{array}$ & $\begin{array}{c}E R R^{\mathrm{a}} \\
(8)\end{array}$ & $\begin{array}{c}\ddot{v}_{\text {pre }} \\
10^{-25} \mathrm{~Hz} \mathrm{~s}^{-2} \\
\text { (9) }\end{array}$ & $\begin{array}{l}n_{\mathrm{obs}} \\
(10)\end{array}$ & $\begin{array}{c}A \\
(11)\end{array}$ \\
\hline $0450-18$ & 5.9 & 5.7 & 0.2 & 0.8 & 4960 & +0.013 & 4 & 0.0060 & 6.5 & -1.48 \\
\hline $0736-40$ & 185.4 & 22.3 & 163.1 & 69.7 & 4880 & -8.389 & 6 & 0.0015 & +16983.1 & -0.01 \\
\hline $0740-28$ & 740.5 & 362.5 & 378.0 & 7.2 & 4930 & -39.334 & 5 & 1.8298 & -64.5 & +0.38 \\
\hline $0835-41$ & 7.7 & 1.5 & 6.2 & 26.2 & 4930 & +0.366 & 9 & 0.0009 & +1238.6 & -1.02 \\
\hline $0959-54$ & 270.9 & 56.2 & 214.7 & 22.6 & 4870 & -13.850 & 3 & 0.0271 & -1535.5 & +0.78 \\
\hline $1054-62$ & 8.8 & 8.3 & 0.5 & 1.4 & 4950 & -0.303 & 8 & 0.0051 & -179.1 & -1.26 \\
\hline $1133+16$ & 1.4 & 0.4 & 1.0 & 22.6 & 5220 & +0.062 & 4 & 0.0002 & +745.5 & -1.64 \\
\hline $1221-63$ & 6.6 & 6.0 & 0.6 & 1.1 & 4950 & -0.096 & 8 & 0.0726 & -4.0 & -1.71 \\
\hline $1240-64$ & 14.2 & 9.4 & 4.8 & 3.1 & 4950 & -0.774 & 3 & 0.0104 & -224.0 & -1.05 \\
\hline $1323-58$ & 79.0 & 63.2 & 15.8 & 1.5 & 4640 & +2.472 & 4 & 0.0029 & +2592.1 & -0.18 \\
\hline $1323-62$ & 48.7 & 30.8 & 17.9 & 1.7 & 4950 & -1.554 & 3 & 0.0719 & -64.8 & -0.38 \\
\hline $1356-60$ & 97.3 & 22.4 & 74.9 & 14.7 & 4660 & +5.048 & 14 & 0.5814 & +26.0 & -0.64 \\
\hline $1358-63$ & 168.2 & 89.1 & 79.1 & 2.4 & 4360 & +8.553 & 6 & 0.0140 & +1827.0 & +0.40 \\
\hline $1426-66$ & 5.6 & 4.8 & 0.8 & 1.6 & 4910 & -0.197 & 3 & 0.0005 & -1244.0 & -1.17 \\
\hline $1449-64$ & 28.6 & 7.4 & 21.2 & 13.4 & 4590 & +1.561 & 10 & 0.0391 & +119.7 & -1.09 \\
\hline $1451-68$ & 4.5 & 4.4 & 0.1 & 1.4 & 4950 & -0.029 & 2 & $<0.0001$ & -5612.5 & -2.02 \\
\hline $1556-44$ & 5.5 & 4.8 & 0.7 & 1.7 & 4920 & +0.217 & 6 & 0.0018 & +354.7 & -1.71 \\
\hline $1557-50$ & 17.7 & 17.1 & 0.6 & 1.0 & 4950 & -0.751 & 7 & 0.1076 & -2.1 & -1.29 \\
\hline $1641-45$ & 147.8 & 102.6 & 45.2 & 1.8 & 4810 & +4.169 & 5 & 0.1288 & +97.1 & +0.04 \\
\hline $1642-03$ & 40.5 & 39.3 & 1.2 & 1.0 & 4900 & -0.299 & 4 & 0.0016 & -549.1 & -0.57 \\
\hline $1706-16$ & 78.8 & 18.2 & 60.6 & 20.4 & 4360 & +6.394 & 9 & 0.0043 & +4497.5 & 0.00 \\
\hline $1727-47$ & 17.0 & 5.6 & 11.4 & 16.8 & 3000 & +2.403 & 3 & 1.4056 & +5.1 & -0.34 \\
\hline $1749-28$ & 42.3 & 39.5 & 2.8 & 1.0 & 4960 & -0.223 & 2 & 0.0111 & -60.2 & -0.45 \\
\hline $1822-09$ & 68.9 & 16.6 & 52.3 & 17.8 & 3590 & +7.364 & 12 & 0.1809 & +122.1 & +0.14 \\
\hline $1929+10$ & 24.0 & 23.2 & 0.8 & 1.5 & 3840 & -1.584 & 7 & 0.0035 & -1376.2 & -0.86 \\
\hline $1933+16$ & 2.7 & 1.9 & 0.8 & 2.4 & 4940 & +0.120 & 2 & 0.0234 & +15.4 & -1.86 \\
\hline $2045-16$ & 0.8 & 0.7 & 0.1 & 1.1 & 4960 & +0.005 & 9 & 0.0005 & +33.9 & -1.72 \\
\hline
\end{tabular}

a Errors are $2 \sigma$ formal standard errors and refer to the last significant digit.

third-order polynomial fits to the data $\left(\chi_{23}^{2}=\chi_{2}^{2} / \chi_{3}^{2}\right)$; Col. 6 contains the timespan of the observations; Cols. 7 and 8 give the resulting frequency second time derivative and its associated formal standard error; while Col. 9 gives the value expected from the simple spin-down law $\left(\ddot{v}_{\text {pre }}=n \dot{v}^{2} / v\right)$, assuming $n=3$; Col. 10 lists the observed braking index $\left(n_{\text {obs }}=v \ddot{v}_{\text {obs }} / \dot{v}^{2}\right)$ and Col. 11 gives the timing noise activity parameter $(A)$. The results, among other things, show that the observed frequency second derivative $\left(\ddot{v}_{\text {obs }}\right)$ is highly variable: being negative for 13 pulsars and positive for 14 objects. Furthermore, $\left|\ddot{v}_{\text {obs }}\right|$ in most cases deviates from the expected value of 3 by several orders of magnitude. As a consequence, the inferred braking indices $\left(n_{\text {obs }}\right)$ have values ranging from $\sim-10^{5}$ to $+10^{4}$. This result is consistent with the measured $\ddot{v}_{\text {obs }}$ being severely contaminated by random steps in $\dot{v}$. Assuming that the amplitudes of these steps have nonzero mean (Cordes \& Helfand 1980) and consequently could introduce a positive or negative bias to the observed frequency second derivative $\left(\ddot{v}_{\text {obs }}\right)$, one would expect that $\left|\ddot{v}_{\text {obs }}\right|$ will be strongly correlated with the timing noise statistics $\left(A\right.$ and $\left.\sigma_{\mathrm{R} 23}\right)$.

Figure 1 plots the timing noise statistic $\sigma_{\mathrm{R} 23}$ against (Fig. 1a) the absolute magnitude of the observed frequency second derivative $\left(\left|\ddot{v}_{\text {obs }}\right|\right)$, and (Fig. 1b) the expected frequency second derivative from the standard spin-down model ( $\left.\ddot{v}_{\text {pre }}\right)$, both on the log-log scale. Similarly, Fig. 2a plots the timing noise activity parameter $(A)$ against $\log \left|\ddot{v}_{\text {obs }}\right|$, while Fig. $2 \mathrm{~b}$ plots $A$ against $\ddot{v}_{\text {pre. }}$ As expected, Figs. $1 \mathrm{~b}$ and $2 \mathrm{~b}$ reveal no apparent correlations between the two timing noise statistics and the secular frequency second derivative ( $\left.\ddot{v}_{\text {pre }}\right)$. However, Fig. 1a shows a clearly defined trend in which objects with large $\left|\ddot{v}_{\text {obs }}\right|$ are characterized by large $\sigma_{\mathrm{R} 23}$. Figure $2 \mathrm{a}$ also shows a similar trend between $A$ and $\left|\ddot{v}_{\text {obs }}\right|$. Simple least squares analyses of the current HartRAO data show that the absolute magnitude of the observed frequency second derivative is significantly correlated with the timing noise statistics. In particular, we obtain $r=+0.95$ and +0.90 , respectively, as the correlation coefficients between $\log \sigma_{\mathrm{R} 23}$ and $\log \left|\ddot{v}_{\text {obs }}\right|$ and between $A$ and $\log \left|\ddot{v}_{\text {obs }}\right|$ (where $\ddot{v}_{\text {obs }}$ is in units of $10^{-25} \mathrm{~Hz} / \mathrm{s}^{-2}$ ). On the other hand, similar analysis reveals no appreciable correlation between the timing noise statistics and the predicted frequency second derivative. More quantitatively, we estimated $r \sim 0.01$ and 0.1 as the correlation coefficients between $A$ and $\log \ddot{v}_{\text {pre }}$ and $\log \sigma_{\mathrm{R} 23}$ and $\log \ddot{v}_{\text {pre }}$, respectively.

\section{Discussion}

Significant measurements of the braking index $(n)$ of radio pulsars are essential in order to constrain the mechanism of angular momentum loss from the spinning neutron stars. However, 

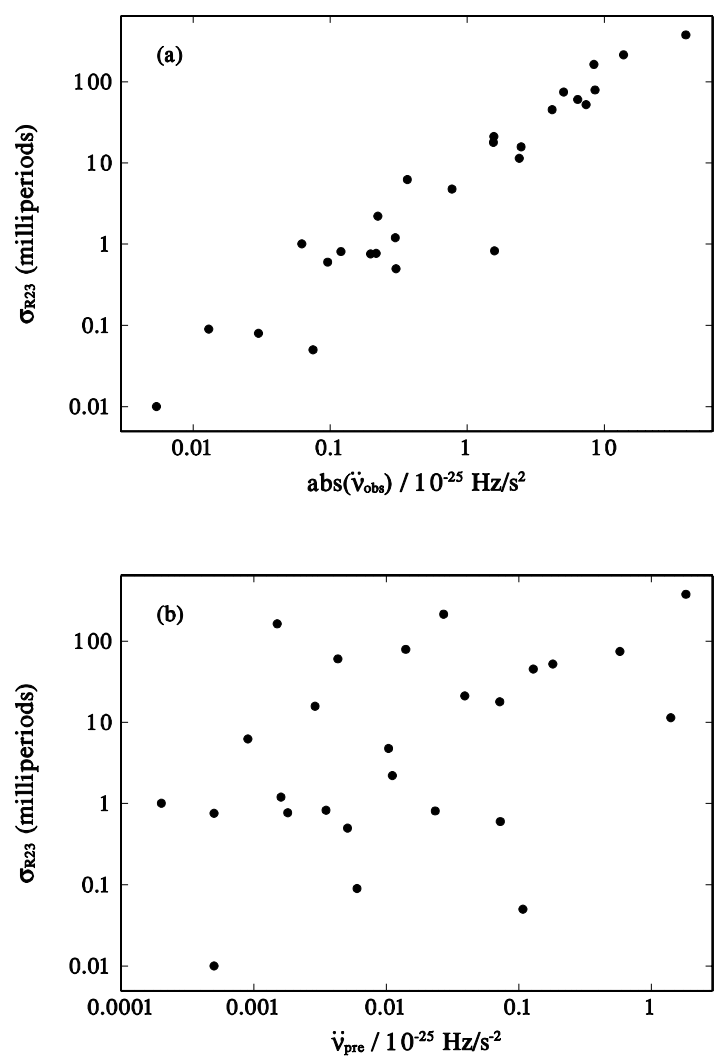

Fig. 1. Plots showing the relationship between the logarithm of the timing noise parameter $\left(\sigma_{\mathrm{R} 23}\right)$, obtained as described in text, and the logarithms of a) the absolute magnitude of the observed frequency second derivative $\left(\left|\ddot{v}_{\text {obs }}\right|\right)$ and $\left.\mathbf{b}\right)$ the model-predicted frequency second derivative $\left(\ddot{v}_{\text {pre }}\right)$ for the current 27 HartRAO pulsars.

after more than 3 decades of timing observations of radio pulsars, $n$ has been significantly measured for only about five pulsars (Manchester et al. 1985; Lyne \& Pritchard 1988; Lyne et al. 1996; Camilo et al. 2000; Zhang et al. 2001). In the context of the standard model, Eq. (1) suggests that the braking index explicitly depends on three pulsar observables - the rotation frequency $(v)$, and its first and second derivatives $(\dot{v}$ and $\ddot{v})$ respectively. While $v$ and $\dot{v}$ are relatively easy to measure (giving timing data that span a few hundreds of days), a number of factors have combined to make significant measurement of $\ddot{v}$ as related to the braking index $n$ in Eq. (2) difficult, or impossible in most cases. For instance, assuming that all isolated pulsars strictly obey the simple spin-down law (Eq. (1)), the expected secular $\ddot{v}_{\text {pre }}$ would be extremely small for most pulsars. For a typical middle-aged radio pulsar characterized by $v=1 \mathrm{~Hz}$ and $\dot{v} \sim 10^{-15} \mathrm{~Hz} / \mathrm{s}$, the expected frequency second derivative $\left(\ddot{v}_{\mathrm{pre}}\right)$ is $\sim 10^{-30} \mathrm{~Hz} / \mathrm{s}^{2}$ (assuming a canonical value of $n=3$ ). Table 1 shows that $10^{-30} \leq \ddot{v}_{\text {pre }} \leq 2 \times 10^{-25}$ for the 27 HartRAO pulsars, which is too small to be significantly measured even with timing data that span some hundreds of years.

However, given the prevalence of timing noise activity in radio pulsars and the fact that the statistics of radio pulsar timing noise have been shown (Cordes \& Downs 1985; Chukwude 2002) to be non-stationary: implying that its effects increase with the time span of observations, it becomes
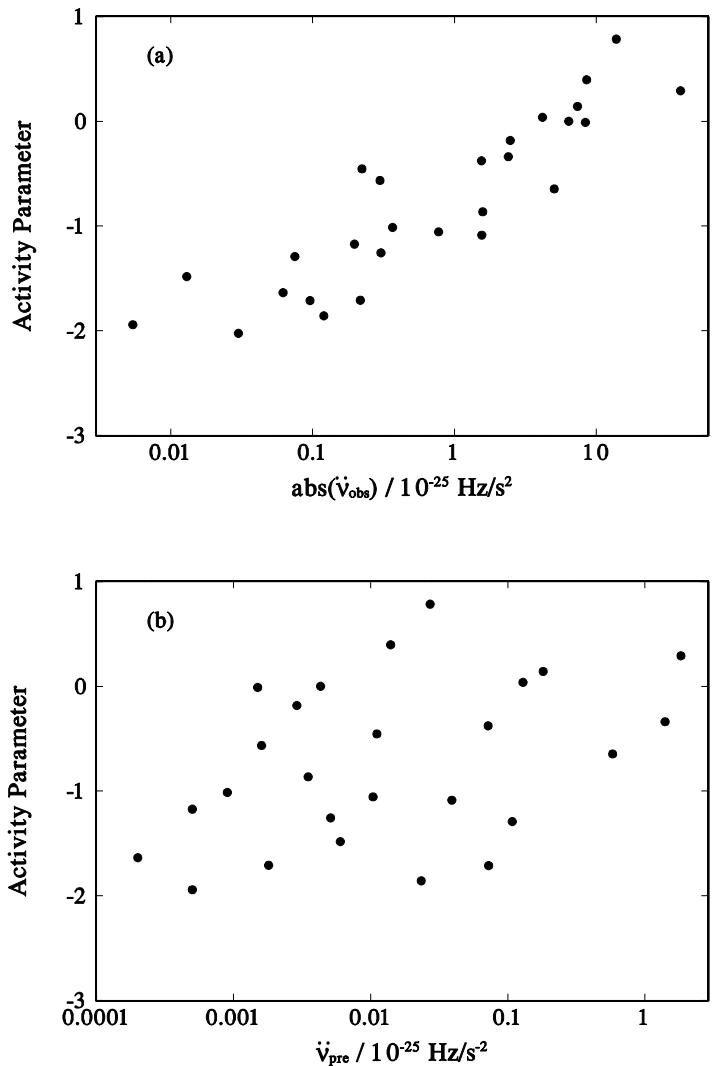

Fig. 2. VPlots showing the relationship between the activity parameter $A$ and the logarithms a) the absolute magnitude of the observed frequency second derivative $\left(\left|\ddot{v}_{\text {obs }}\right|\right)$ and $\left.\mathbf{b}\right)$ the expected frequency second derivative $\left(\ddot{v}_{\text {pre }}\right)$ for the current HartRAO pulsars.

difficult to see how such a long span of observations could really help in measuring these extremely small systematic $\ddot{v}$ 's. The prevailing picture is that timing noise fluctuations in the pulsar rotation rates seriously contaminate the observed coefficient of the cubic term, $\ddot{v}_{\text {obs }}$, in most pulsars. In particular, Cordes \& Helfand (1980) have noted that timing noise activitydominated $\ddot{v}$ (i.e. $\ddot{v}_{\mathrm{obs}} \approx \ddot{v}_{\text {tno }}$ ) would be random in nature, with either sign, and would be characterized by $\left|\ddot{v}_{\text {obs }}\right| \ddot{v}_{\text {pre }} \mid>1$. Our results show that the observed frequency second derivative ( $\ddot{v}_{\text {obs }}$ ) for the current HartRAO sample is positive for 14 pulsars and negative for 13 pulsars and that $1<\left|\ddot{v}_{\text {obs }} / \ddot{v}_{\text {pre }}\right|<$ $10^{5}$. Accordingly, the inferred braking indices vary over a wide range, $-10^{4}<n_{\mathrm{obs}} \leq+10^{5}$. Physical interpretation of this result is not immediately obvious. Even the moderate positive braking indices of 6.5 and 5.1 obtained, respectively, for the pulsars B0450-18 and B1727-47 are still too high to be associated to pulsar torque braking processes, unless the $\ddot{v}_{\text {obs }}$ is significantly contaminated by recovery of $\dot{v}$ from unresolved glitch events. Even though anomalous positive braking indices observed in a few young/middle-aged pulsars (Shemar \& Lyne 1996) have been plausibly explained as characteristics of interglitch recovery in these objects (Alpar 1998), negative braking indices are still difficult to understand in context of the prevailing picture of radio pulsar spin evolution. Using a method that negates the measurement of frequency second derivative, Johnston \& Galloway (1999) have 
argued that large negative braking indices could be attributed to glitches occurring in between the epochs of the timing solution. However, no resolved glitch events have been reported in the present data, suggesting that the speculated influence of glitches on the observed braking indices is minimal at best.

A much more plausible interpretation of the current results can be obtained if one assumes that the observed frequency second derivatives $\left(\ddot{v}_{\text {obs }}\right)$, at least for the current data, are composed of two components: a secular component $\left(\ddot{v}_{\text {pre }}\right)$ and a component arising from an accumulation of fluctuations in the pulsar spin rates $\left(\ddot{v}_{\text {tno }}\right)$. The most probable scenario is that the timing noise component strongly dominates over the secular component (i.e. $\left|\ddot{v}_{\text {tno }}\right| \gg \ddot{v}_{\text {pre }}$ ), hence it is reasonable to argue that the $\ddot{v}_{\text {obs }}$ listed in Table 1 are merely manifestations of timing noise activity rather than due to an intrinsic pulsar torque braking process.

Perhaps the strongest observational evidence for the timing noise interpretation of the $\ddot{v}_{\text {obs }}$ could be found in the observed strong correlations between the two timing noise statistics the activity parameter $A$ (a qualitative measure of the fluctuations in the pulsar rotation rates due to timing noise activity) and $\sigma_{\mathrm{R} 23}$ (a measure of the amount of timing noise absorbed by the coefficient of cubic the term) - and $\left|\ddot{v}_{\text {obs }}\right|$. In principle, if the large $\sigma_{\mathrm{R}}(2, T)$ are mainly due to the inadequacy of a secondorder model and a third-order model simply provides a better description of the intrinsic pulsar rotational history, one would expect no significant correlation between $\sigma_{\mathrm{R} 23}$ and the resulting coefficient of the cubic term ( $\left.\ddot{v}_{\text {obs }}\right)$, as was observed for $\ddot{v}_{\text {pre }}$. However, the fact that there exists a significant correlation, $r \sim+0.95$, between them suggests that the $\ddot{v}_{\text {obs }}$, even if it contains a secular component, is strongly dominated by the timing noise components in the data. Hence, as would be expected, the larger the amount of fluctuations absorbed by the cubic polynomial $\left(\sigma_{\mathrm{R} 23}\right)$ the more the absolute magnitudes of the resultant $\ddot{v}_{\text {obs }}$ are biased to higher values. Similarly, the equally strong correlation between $A$ and $\left|\ddot{v}_{\text {obs }}\right|$ further supports the timing noise interpretation of the observed frequency second derivatives. The $A-\log \left|\ddot{v}_{\text {obs }}\right|$ correlation suggests that pulsars characterized by higher level of timing noise activity, on average, have larger absolute values of frequency second derivative.

The obvious implication of the current results is that the secular braking index, even when it exists, may never be measurable for most pulsars (especially those with moderate and small values of $\dot{v}$ ) because of the strong dominant effect of rotational fluctuations in the form of timing noise activity. Moreover, the argument that significant measurements of $\ddot{v}$ for middle-aged and old pulsars would require a very long timespan of observations appears weakened by the observed correlations. As a consequence, future efforts at measuring the braking indices of radio pulsars, especially methods that aim at minimizing the effect of timing noise, would have to explore alternative techniques to the conventional phase-connected solution of observations spanning decades in time. However, phase-connected solutions of timing data could still be important in measuring systematic frequency second time derivative if there is a realistic method of modelling out the timing noise component from the secular component of $\ddot{v}$.

\section{Conclusion}

We have investigated the statistical implication of long-term intrinsic fluctuations in the rotation of radio pulsars for the observed braking index. The result reveals that the observed frequency second derivative is strongly correlated with the statistics used to quantify fluctuations of timing noise origin. This, most probably, suggests that significant measurement of the secular component of the frequency second derivative in the presence of radio pulsar timing noise could be very difficult, if not impossible, for most (especially the old and middle-aged) pulsars owing to the dominant presence of the timing noise component of $\ddot{v}$.

Acknowledgements. I am very grateful to G. D. Nicolson, the director of HartRAO, and C. S. Flanagan for providing me with the pulsar data, J. O. Urama and A. A. Ubachukwu for very helpful discussions, M. J. Gaylard for critically reading through the manuscript and an anonymous referee for useful comments which helped to improve the paper. This work was done while the author was in HartRAO as a visiting research student and he has the pleasure to acknowledge the support and hospitality of HartRAO staff. IAU Commission 38 supported the visit to South Africa with a travel grant.

\section{References}

Alpar, M. A. 1998, Adv. Space. Res., 21, 159

Baykal, A., Alpar M. A., Boynton, P. E., \& Deeter, J. E. 1999, MNRAS, 306, 207

Boynton, P. E., Groth, E. J., Hutchinson, D. P., et al. 1972, ApJ, 175, 217

Camilo, F., Kaspi, V. M., Lyne, A. G., et al. ApJ, 541, 367

Chukwude, A. E. 2002, Ph.D. Thesis, University of Nigeria, Nsukka Cordes, J. M. 1993, in Planets Around Pulsars, ed. J. A. Phillips, S. E. Thorsett, \& S. R. Kulkarni (San Francisco: ASP), ASP Conf. Ser., 36, 43

Cordes, J. M. 1980, ApJ, 237, 216

Cordes, J. M., \& Downs, G. S. 1985, ApJS, 59, 343

Cordes, J. M., \& Helfand, D. J. 1980, ApJ, 239, 640

Cordes, J. M., \& Greenstein, G. 1981, ApJ, 245, 1060

D’Alessandro, F., McCulloch, P. M., Hamilton, P. A., \&

Deshpande, A. A. 1995, MNRAS, 277, 1033

D'Alessandro, F., McCulloch, P. M., Hamilton, P. A., \& McConnell, D. 1993, MNRAS, 261, 883

Goldreich, P., \& Julian, W. H. 1969, ApJ, 157, 869

Flanagan, C. S. 1995, Ph.D. Thesis, Rhodes University, Grahamstown, South Africa

Groth, E. J. 1975, ApJS, 29, 443

Lyne, A. G., \& Graham-Smith, F. 1998, Pulsar Astronomy (Cambridge University Press)

Lyne, A. G., \& Pritchard, R. S. 1988, MNRAS, 233, 667

Lyne, A. G., Pritchard, R. S., Graham-Smith, F., \& Camilo, F. 1996,

Nature, 381, 497

Manchester, R. N., Durdin, J. M., \& Newton, L. M. 1985, Nature, 313,31

Manchester, R. N., \& Taylor, J. H. 1977, Pulsars (San Francisco: Freeman)

Shemar, A. L., \& Lyne, A. G. 1996, MNRAS, 282, 677

Wang, N., Manchester, R. N., Pace, R. T. et al. 2000, MNRAS, 317, 843

Zhang, W., Marshall, F. E., Gotthelf, E. V., Middleditch, J., \& Wang, Q. D. 2001, ApJ, 554, L177 